\title{
YANG-MILLS VACUA IN LANDAU GAUGE *
}

Paul H. Frampton, Teruhiko Hagiwara, William F. Palmer and Stephen S. Pinsky

Department of Physics

The Ohio State University

Columbus, Ohio 43210

\section{ABSTRACT}

A vacuum gauge field $A_{\mu}^{a}$ for Yang-Mills theory is constructed; this field (i) is pure vacuum $\left(A_{\mu}^{a}=0\right.$ ) at the origin, (ii) approaches at large distances, the Belavin-Polyakov-Schwartz-Tyupkin pseudoparticle, and (iii) satisfies $\partial_{\mu} \mathrm{A}_{\mu}^{\mathrm{a}}=0$ everywhere. The net topological charge is zero, and there is a Dirac-like string terminating at the origin.

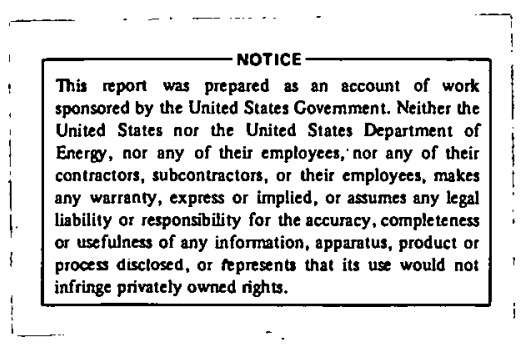

* Supported in part by the U.S. Department of Energy under contract number EY-76-C-02-1545.*000. 


\section{DISCLAIMER}

This report was prepared as an account of work sponsored by an agency of the United States Government. Neither the United States Government nor any agency Thereof, nor any of their employees, makes any warranty, express or implied, or assumes any legal liability or responsibility for the accuracy, completeness, or usefulness of any information, apparatus, product, or process disclosed, or represents that its use would not infringe privately owned rights. Reference herein to any specific commercial product, process, or service by trade name, trademark, manufacturer, or otherwise does not necessarily constitute or imply its endorsement, recommendation, or favoring by the United States Government or any agency thereof. The views and opinions of authors expressed herein do not necessarily state or reflect those of the United States Government or any agency thereof. 


\section{DISCLAIMER}

Portions of this document may be illegible in electronic image products. Images are produced from the best available original document. 
Because the physics of quantum chromodynamics ${ }^{1}$ is evidently very different from that of quantum electrodynamics, any theoretical distinction between non-abelian and abelian gauge field theories arouses great interest. One such distinction, the full significance of which is yet to be established, was pointed out recently by Gribov. ${ }^{2}$ The fundamental observation is that, unlike the case of electrodynamics, the imposition of a continuous gauge condition together with appropriate boundary conditions is not sufficient in a Yang-Mills theory to specify uniquely the gauge field. In particular, the Coulomb gauge was studied in Ref.2.

In the present Letter, we study the vacuum of a Yang-Mills theory, but now in the Landau gauge. The situation is superficially similar to that of the Coulomb gauge but there are significant and important differences. For example, the topological properties of the Landau gauge are quite different, as will become clear below.

The SU(2) theory we are considering has lagrangian

$$
\begin{aligned}
\mathcal{L} & =\frac{1}{4} F_{\mu \nu}^{a} F_{\mu \nu}^{a}: \\
F_{\mu \nu}^{a} & =\partial_{\mu} A_{\nu}^{a}-\partial_{\nu} A_{\mu}^{a}+g \epsilon^{a b c} \dot{A}_{\mu}^{b} A_{\nu}^{c}
\end{aligned}
$$

with $a=1,2,3$. Let us consider the general gauge transformation

$$
U(x)=z_{4}+\underline{i} \underline{\sigma} \cdot \underline{z}
$$

where $z_{\mu}(x)$ is a real unit vector. If we perform such a gauge transformation on the pure vacuum $A_{\mu}^{a}=0$ we arrive at the field

$$
\mathrm{A}_{\mu}^{\mathrm{a}}=\frac{2}{\mathrm{~g}} \eta_{\alpha \beta}^{\mathrm{a}}\left(\partial_{\mu} z_{\alpha}\right) z_{\beta}
$$

where $\eta_{\alpha \beta}^{a}$ is the symbol having the properties ${ }^{3}$

$$
\begin{aligned}
& \eta_{b c}^{a}=\epsilon_{a b c} \quad a, b, c=1,2,3 \\
& \eta_{b 4}^{a}=-\eta_{4 b}^{a}=\delta_{a b} .
\end{aligned}
$$


For example, choosing $z_{\mu}=\hat{x}_{\mu}$ we arrive at

$$
A_{\mu \cdot}^{a}=\frac{2}{g} \frac{\eta_{\mu \beta}^{a} x_{\beta}}{R^{2}}
$$

where $R^{2}=x_{4}^{2}+\underline{x}^{2}$. This is the zero-size-1imit of the pseudoparticle solution of Belavin et al. ${ }^{4}$ (BPST). Note that throughout we are considering only such classical solutions in euclidean space.

As a specific ansatz, for which we shall claim a vacuum copy exists, we parametrize $z_{\mu}$ as follows

$$
z_{\mu}=\left(\begin{array}{c}
\cos g(R, \alpha) \\
\hat{x} \sin g(R, \alpha)
\end{array}\right)
$$

where $\alpha=\tan ^{-1}\left(r / x_{4}\right), \underline{\hat{x}}=\underline{x} / r, r^{2}=\underline{x}^{2}$.

The boundary conditions to be imposed are

$$
\begin{array}{lll}
\mathrm{g}(\mathrm{R}, \alpha) \stackrel{\mathrm{R} \rightarrow \infty}{\longrightarrow} \alpha & 0 \leq \alpha<\pi \\
\mathrm{g}(\mathrm{R}, \alpha) \stackrel{\mathrm{R} \rightarrow 0}{\longrightarrow} 0 & 0 \leq \alpha \leq \pi .
\end{array}
$$

Thus, there is pure vacuum at the origin, and at large distances, for all $\alpha$ except $\alpha=\pi$, there is the limit of BPST, Eq. (7).

, We further insist that $F_{\mu \nu}^{a}=0$ everywhere, with $F_{\mu \nu}^{a}$ given by Eq. (2).

The negative $x_{4}$-axis $(\alpha=\pi)$, excluded in Eq. (9), is singular and requires separate discussion related to topological charge conservation. Now impose the Landau gauge condition $\partial_{\mu} A_{\mu}^{a}=0$ which, from Eq. (4), implies

$$
\eta_{\alpha \beta}^{a}\left(\mathbf{u}_{\alpha}^{\prime}\right) z_{\beta}=0
$$

Inserting the ansatz, Eq.(8), we find after some algebra 


$$
g=\frac{\sin 2 g}{R^{2} \sin ^{2} \alpha}
$$

Note that in general the copy of any field configuration $A_{\mu}^{\prime}$ in the Landau gauge can be obtained as an extremum of the action

$$
S=\int d^{4} x \operatorname{Tr}\left[\partial_{\mu} U^{\dagger} \partial_{\mu} U-2 \partial_{\mu} U U^{\dagger} A_{\mu}^{\prime}\right]
$$

To analyze the required solution of Eq.(12) for $\alpha$ in the vicinity of $\alpha=0, \frac{\pi}{2}, \pi$ it is convenient to use variables $t=\ln R$ and $g(R, \alpha)=\alpha f(t, \alpha)$ whereupon one finds

$$
\ddot{\mathrm{f}}+2 \dot{\mathrm{f}}+\left[\frac{\partial^{2}}{\partial \alpha^{2}}+2\left(\frac{1}{\alpha}+\cot \alpha\right) \frac{\partial}{\partial \alpha}\right] \mathrm{f}+\frac{2 \cot \alpha}{\alpha} \mathrm{f}=\frac{\sin (2 \alpha \mathrm{f})}{\alpha \sin ^{2} \alpha}
$$

Assuming sufficient smoothiness in $\alpha$ for all t (i.e., neglecting $\alpha$ derivatives), as special cases of Eq.(14) one may cite the mechanical analogs at fixed $\alpha$ :

(i) $\alpha \ll 1$ gives

$$
\ddot{\mathrm{f}}+2 \dot{\mathrm{f}}=\frac{4}{3} \mathrm{f}\left(1-\mathrm{f}^{2}\right)
$$

corresponding to attenuated motion in the potential

$$
U(f)=\frac{1}{3}\left(1-f^{2}\right)^{2}
$$

satisfying the required boundary conditions $f=0$ at $t=-\infty$ and $f=1$ at $t \rightarrow+\infty$.

(ii) $\alpha=\frac{\pi}{2}-\beta, \beta \ll 1$ yields

$$
\ddot{\mathrm{f}}+2 \dot{\mathrm{f}}=\frac{2}{\pi} \sin \pi \mathrm{f}
$$

which describes dissipative particle motion in the potential well

$$
U(f)=\frac{2}{\pi^{2}} \cos \pi f
$$

which again is seen to be consistent with the given boundary conditions. (iii) Consider now $\alpha=\pi-\beta, \beta<1$. This gives 


$$
\ddot{\mathrm{f}}+2 \dot{\mathrm{f}}=\frac{1}{\pi \beta^{2}} \sin 2 \pi \mathrm{f}
$$

corresponding to damped motion in potential

$$
U(f)=\frac{1}{2 \pi^{2} \beta^{2}} \cos (2 \pi f) \text {. }
$$

For $\beta \neq 0$ this is consistent with $\mathrm{f} \rightarrow 0,1$ for $t \rightarrow-\infty,+\infty$, respectively. For $\beta=0$ (or $\alpha=\pi$ ) exactly, only $f(t, \pi)=0$ is allowed as a solution.

For the case of general $\alpha$ we introduce variables $\ell=\sin ^{2} \alpha$ and $\xi=x_{4} / x$ to find

$$
\ddot{g}+2 \dot{g}=\frac{\sin 2 g}{\ell}-\frac{1}{\ell^{2}} \frac{\partial^{2} g}{\partial \xi^{2}}
$$

The form, Eq. (21), is useful because it enables us to make the following quasi-mechanical analog in continuum limit. An infinite ensemble of pendula is arranged to pivot around a horizontal $\xi$-axis. The pendulum lengths range from $l=1$ (at $\xi=0$ ) to $l=0$ (at $\xi=+\infty$ ) according to $l=\left(1+\xi^{2}\right)^{-1}$. pendula start at $t=-\infty$ in a vertical position; thereafter, for $0 \leq \alpha \leq \pi / 2$, $2 \mathrm{~g}(\mathrm{R}, \alpha)$ is the angle from the vertical of the pendulum at $\xi=\cot \alpha$ for time $t=\ln R$. For $\pi / 2 \leq \alpha<\pi$, we have $g(\alpha, R)=\pi-g(\pi-\alpha, R)$. To reproduce the final term in $\mathrm{Eq} .(20)$ requires the pendula to be coupled by tension springs with negative (but constant) torsional moduli. The system then moves such that for all $\xi, t$ one has $\partial g / \partial \xi<0$ and $\partial^{2} g / \partial \xi^{2}>0$. Thus the final term in Eq. (21) always acts to decrease $g$. This term is infinitely large for $\ell \rightarrow 0$ and hence $g(R, 0)=0$ for all $R$; the same term becomes progressively less important for $\xi \rightarrow 0$ where the motion is that of a free damped pendulum. This mechanical system comes to rest for $t \rightarrow \infty$ such that

$$
\ell \sin 2 g=\partial^{2} g / \partial \xi^{2}
$$

which gives $g(\infty, \alpha)=\alpha$, as required. Also, the smoothness assumption mentioned 
immediately prior to Eq.(15) is seen to be consistent with this result. We should emphasize that we have been unable to demonstrate rigorously that the above motion is possible for the mechanical analog, but every consistency check we have made is positive.

Now we turn to the most subtle part of the analysis. Our vacuum copy becomes, for $\mathrm{R} \rightarrow \infty$, the BPST form, Eq. (7), and the reader may well ask why the topologica1 charge

$$
Q=\frac{g^{2}}{32 \pi^{2}} \int d^{4} \times F_{\mu \nu}^{a} \tilde{F}_{\mu \nu}^{a}
$$

should not have, therefore, the value $Q=1$ which would contradict our assertion that $\dot{\mathrm{F}}_{\mu \nu}^{\mathrm{a}}=0$ everywhere.

To understand how we have $Q=0$ it is profitable to consider the quantity

$$
q\left(x_{4}\right)=\frac{g^{2}}{96 \pi^{2}} \int_{\substack{\text { fixed } \\ x_{4}}} d^{3} x \varepsilon^{i j k} \epsilon_{a b c} A_{i}^{a} A_{j}^{b} A_{k}^{c}
$$

This measures the amount of topological flux crossing a fixed-x slice $_{4}$ of euclidean 4-space. The total charge will then be given by

$$
Q=q(+\infty)-q(-\infty)
$$

provided, as is the case here, there is no leakage for $r \rightarrow \infty$.

Let us first consider the case where $A_{\mu}^{a}$ is given everywhere by Eq. (7). Then one has $Q=+1$ since

$$
F_{\mu \nu}^{a} \tilde{F}_{\mu \nu}^{a}=\frac{32 \pi^{2}}{g^{2}} \delta^{4}(x)
$$

and

$$
q\left(x_{4}\right)=\frac{2 x_{4}}{\pi} \int_{0}^{\infty} d r \frac{r^{2}}{\left(r^{2}+x_{4}^{2}\right)^{2}}=\frac{x_{4}}{2\left|x_{4}\right|}
$$


This may also be written (as before, $\alpha=\tan ^{-1} r / x_{4}$ )

$$
\begin{aligned}
q\left(x_{4}\right) & =\left.\frac{1}{2 \pi^{2}} \int d^{3} x \frac{\sin ^{2} \alpha}{r^{2}} \frac{d \alpha}{d r}\right|_{x_{4}} \text { fixed } \\
& =\frac{1}{\pi}\left[\alpha\left(r=\infty, x_{4}\right)-\alpha\left(r=0, x_{4}\right)\right]
\end{aligned}
$$

which agrees with Eq.(27) since $q( \pm \infty)= \pm 1 / 2$ and hence, from Eq.(25), $Q=+1$.

In the present case, after some:algebra one finds a formula different from Eq. (29), namely

$$
q\left(x_{4}\right)=\frac{1}{\pi}\left[g\left(r=\infty, x_{4}\right)-g\left(r=0, x_{4}\right)\right]
$$

For $x_{4}>0$, the result coincides with Eq. (29) for large $x_{4} \cdot$ For negative $x_{4}$, one must now write

$$
g\left(r, x_{4}\right)=f\left(r, x_{4}\right) \propto\left(r, x_{4}\right)
$$

and observe that because of the singular behavior already noted for Eq. (19) one has

$$
f\left(x=0, x_{4}\right)=0, x_{4}<0
$$

Thus, from Eq. (30), one obtains that $q( \pm \infty)=+1 / 2$ and therefore from Eq. (25), $Q=0$ consistent with $F_{\mu \nu}^{a} \equiv 0$.

The situation is the following: for $x_{4} \rightarrow-\infty, q\left(x_{4}\right)$ has two contributions; one is $-1 / 2$ from the BPST component, the other is +1 from a string of topological flux along the negative $x_{4}$-axis terminating at the origin.

The necessity for this string is quite analogous to that for the Dirac magnetic monopole ${ }^{5}$ in electrodynamics, where a string of magnetic flux is required to comply with Gauss' theorem.

We speculate that there is a related stringless copy with a different set of boundary conditions, namely pseudoparticle behavior in the positive time hemihypersphere and anti-pseudoparticle behavior in the negative time 
hemihypersphere at $R=\infty$. This solution is necessarily discontinuous on the great equatorial hypercircle and has, of course, zero topological flux. One can imagine a flux conserving deformation of this solution in which the equator is shrunk to increasing latitude circles which finally form the opening of a tube of pinched incoming flux along the negative time axis which cancels the outgoing flux on the rest of the hypersphere. 


\section{REFERENCES}

1. H. Fritzsch et al., Phys. Lett. 47B, 365 (1973);

See also: D. J. Gross and F. Wilczek, Phys. Rev. D 으, 3633 (1973);

S. Weinberg, Phys. Rev. Lett. 31,494 (1973).

2. V. N. Gribov, Lecture at the 12th Winter School of the Leningrad Nuclear Physics Institute, 1977. SLAC-TRANS-176 (1977).

3. G. 't Hooft, Phys. Rev. D 14, 3432 (1976).

4. A. A. Belavin, A. M. Polyakov, A. S. Schwartz and Yu. S. Tyupkin, Phys. Lett. 59B, 85 (1975).

5. P. A. M. Dirac, Proc. Roy. Soc. A133, 60 (1931); Phys. Rev. 74,817 (1948). 